\title{
A novel arch-shaped hybrid composite triboelectric generator using carbon fiber reinforced polymers
}

\author{
Claire-Lise Oney ${ }^{[a]}$, Théo Outrequin ${ }^{[a]}$, Cédric Hubert ${ }^{[a]}$, Xue Yan ${ }^{[a, b]}$, Mustafa Arafa ${ }^{[c]}$, Nicholas \\ Gathercole $^{[a]}$, Chris Rhys Bowen ${ }^{[a]}$
}

\begin{abstract}
With the diminution of the energy sources and the need for using cleaner energy, alternatives must be found. In addition, the desire for energy sources to supply autonomous low-power electronics has led to interest in triboelectric materials. We have fabricated an arch-shaped hybrid carbon fiber reinforced polymer (CFRP) composite based triboelectric device employing a curved upper copper electrode and a flat lower electrode of a polyimide that are both combined with CFRP materials. This device aims to be used as a triboelectric harvesting energy source for self-powered sensors that can be combined with fibre reinforced composite based structures We have been able to produce a voltage up to $300 \mathrm{mV}$ which can charge a capacitor to $250 \mathrm{mV}$. The ability to combine triboelectric and CFRP materials provides a new approach to integrate energy harvesting into engineering structures and manufacture robust harvesting devices.
\end{abstract}

\section{Introduction}

Today, an increasing number of devices and sensors operate in a wireless manner. The total number of connected devices ('Internet of Things') is expected to be 80 billion by 2025, with application ranging from healthcare, transport, cities, and agriculture. [1]. However, such connected sensors often use batteries as a source of energy. As a result, triboelectric energy harvesters have attracted interest as a solution to avoid the use of batteries to mitigate the need for their disposal and management. Triboelectric energy harvesters are of interest since they can convert mechanical energy into electrical energy as a result of contact between two dissimilar materials [2]. The energy produced can be collected and accumulated or directly used to power a sensor (such as temperature, humidity, light or acceleration) or other low-power electronic system. Four different modes have been studied to harvest energy using triboelectric technology [2], these include the: (i) vertical contact-separation mode, (ii) contact sliding mode, (iii) single electrode mode, and (iv) freestanding triboelectric layer mode. In each case, the structure requires two different materials, conductive or insulating, to ensure the exchange of electrons to produce energy. In the contact-separation mode, mechanical movements enable the contrasting surfaces to be in contact or slide against each other

\footnotetext{
[a] Department of Mechanical Engineering, University of Bath, Bath, BA2 7AY; E-mail: c.r.bowen@bath.ac.uk

[b] Science and Technology on Advanced Functional Composites Laboratory, Aerospace Research Institute of Material and Processing Technology, Beijing, 100076, People's Republic of China; E-mail: x_yan@outlook.com

[c] Mechanical Engineering Department, American University in Cairo, Egypt
}

and therefore create an electric charge. The basic mechanism of the vertical contact-separation mode is outlined in Fig. 1.

To facilitate rapid contact and subsequent separation, numerous research groups have considered arch-shaped triboelectric nanogenerators $[3,4,5,6,7,8,9,10]$. However, they often employ a metal and a polymer, and relatively low strength and stiffness materials. However, limited work has attempted to combine triboelectric generators with advanced structural composite materials, such as carbon fiber reinforced polymers (CFRP). The aim of this work is to integrate triboelectric materials with a high strength structural material with the potential to be integrated in an entire structure or unit. This could enable the integration of triboelectric harvesting systems in vehicles (car, train, or plane ...) or other structures (e.g. buildings, bridges ...), where the vibrations producing the desired strain can achieve contact separations events.

We have deliberately selected CFRP as the structural material in this work since an intriguing characteristic of using CFRP laminates is the potential to exploit the anisotropic thermal expansion of the composite to tailor the laminate lay-up and carbon fiber orientation to achieve the desired arch-shape and mechanical properties of the triboelectric generator. The use of anisotropic thermal expansion in CFRP has been used in the design of smart structures [11] and piezoelectric based harvesters [12], but has yet to be undertaken on triboelectric materials. This is not possible with isotropic materials which are often used in triboelectric devices $[3,4,5,6,7,8,9,10]$. This paper aims to present and describe an arch-shaped device composed of CFRP, using copper and polyimide as the triboelectric materials for energy generation.

Figure 1. Schematic of the vertical contact-separating mode principle.

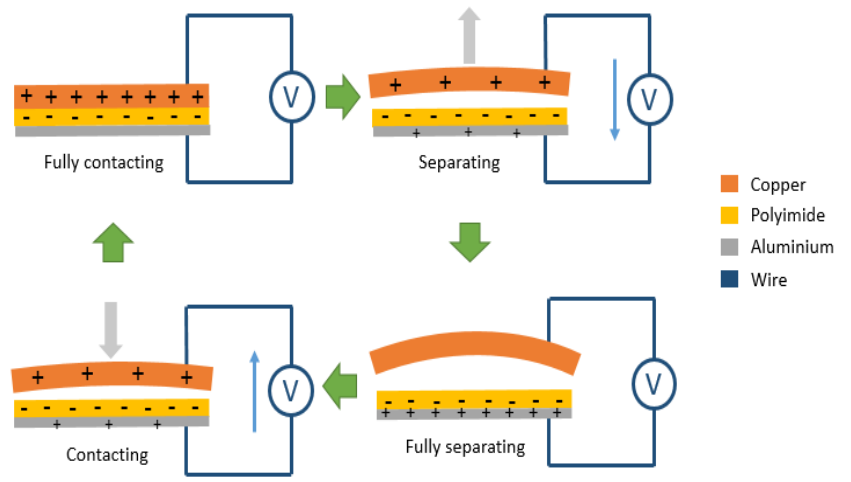

\section{Evaluation of CFRP-triboelectric hybrids}

\section{Control test of initial triboelectric-materials}

The first objective, as a control, was to examine the behavior of the initial triboelectric materials that were selected for integration into CFRP. This involved examination and characterisation of polyimide and copper using the vertical contact-separating mode. Based on the work of Z.L. Wang et al. $[13,14]$ we created simple flat samples to test the effect, to 
compare with the materials after integration with the CFRP structure. This was considered since CFRP production involves autoclaving at elevated temperatures at $\sim 180^{\circ} \mathrm{C}$ and it would be of interest to examine if such a processing temperature subsequently influences the triboelectric behavior. Acrylic plates were used as mechanical substrates and covered by two materials selected. This included copper (thickness of $100 \mu \mathrm{m}$ ) and a polyimide (thickness of $50 \mu \mathrm{m}$ ). The materials have been chosen according to the triboelectric series [13] which ranks materials with their tendency to loose or gain electrons based on their polarity. They are ranked in such a way the copper is more positively charged than the Kapton (polyimide). Since copper layer is naturally conductive it did not require any electrode. Commercially available polyimide sheets (Kapton) were already composed of a thin layer of aluminum $(30 \mathrm{~nm})$ which acted as an electrode.

To charge a capacitor, a direct current is needed. To convert the alternating current $(\mathrm{AC})$ generated by the triboelectric mechanism during contact separation into a direct current (DC) a full wave rectifier was used. Then, the copper and polyimide materials were contacted and separated against one another by hand. The maximum output voltage obtained being close to $0.8 \mathrm{~V}$, see Fig.2.a. Moreover, the system was able to charge a capacitor of $0.1 \mu \mathrm{F}$ up to $15 \mathrm{~V}$ in 30 s; see Fig.2.b.
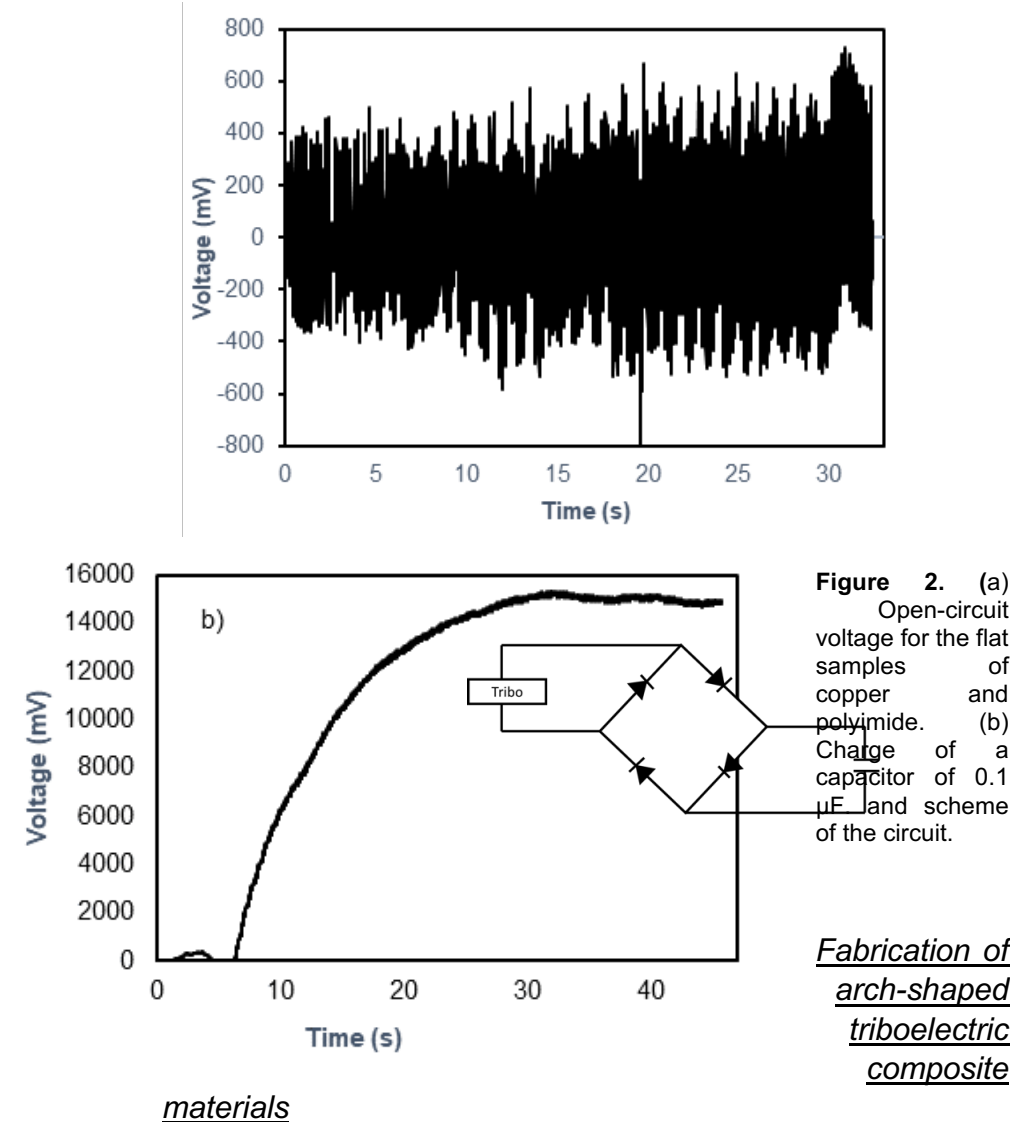

The aim of the work is to demonstrate the co-manufacture of triboelectric materials and CFRP to produce an arc-shaped triboelectric device, and to fabricate a single piece device composed of CFRP as a structural material. The upper layer of the triboelectric harvester is curved to act like a spring to achieve contact separation for triboelectric generation; as in Fig.1. Therefore, the next stage was to study the behaviour of the different triboelectric materials once in contact with carbon fibre reinforced polymers and the influence of temperature and thermal expansion when autoclaved at $180^{\circ} \mathrm{C}$.

To estimate the curvature of the Cu-CFRP plate during curing, the model developed by Timoshenko [15] pertaining to the analysis of bi-metallic strips can be employed. The formulation, which is based on the effective properties of the constituting layers, predicts the curvature due to thermal expansion mismatch as:

$$
\frac{1}{\rho}=\frac{6\left(\alpha_{2}-\alpha_{1}\right)(\Delta T)(1+m)^{2}}{h\left[3(1+m)^{2}+(1+m n)\left(m^{2}+\frac{1}{m n}\right)\right]}
$$

Where is the radius of curvature, $a_{2}$ and $a_{1}$ are the coefficients of thermal expansion of the two layers, $\Delta T$ is the temperature change, $h$ is the total thickness of the plate, and the constants $m$ and $n$ are given by:

$m=\frac{h_{1}}{h_{2}} \quad, \quad n=\frac{E_{1}}{E_{2}}$

with $h$ being the thickness of the individual layer and $E$ being Young's modulus. Accordingly, the deflection at midpoint is given by:

$$
\delta=\frac{l^{2}}{8 \rho}
$$

where $I$ is the length of the plate. A predicted deflection of 11.4 $\mathrm{mm}$ for a plate consisting of a copper layer and a CFRP, each having thickness of $0.1 \mathrm{~mm}$ and a length of $75 \mathrm{~mm}$ when subjected to a change in temperature of $160^{\circ} \mathrm{C}$.

To manufacture the triboelectric-CFRP hybrids unidirectional carbon fibre pre-impregnated with $135 \mathrm{gr} / \mathrm{m}^{2}$ was used. Sandpaper was used to roughen the copper electrode surface to facilitate contact and adhesion with the carbon fibre during the process. Then, one layer of CFRP was applied on a flat sample of copper. The Cu-CFRP samples were placed into an oven at $30^{\circ} \mathrm{C}$ for 3 hours to improve adhesion between the copper and the CFRP. Finally, the composites were placed into an autoclave at a temperature of $180^{\circ} \mathrm{C}$ and a pressure of 7 bar for 3 hours. As predicted, we obtained arch samples of Cu/CFRP composite, the copper being in the inner side of the curve (Fig.3). The experimental bending obtained showed a deflection around 9.98 $\mathrm{mm}$ with a relative error of $9.9 \%$ between model and experiment. Potential reasons for the difference can be changes in stiffness and expansion with temperature [16]. 


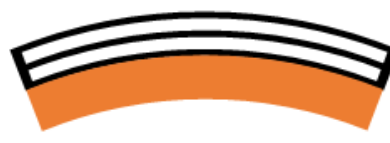

- CFRP

\section{Copper}

(a)

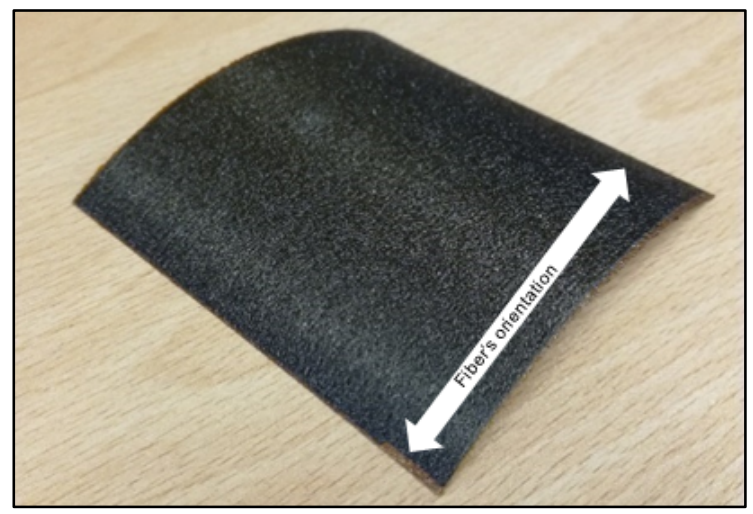

(b)

Figure 3. (a) Schematic showing the fibers' orientation in relation to the curvature. (b) Image of the curved Cu-CFRP combination where sample width is $\sim 75 \mathrm{~mm}$.

Since the copper triboelectric has a larger CTE than CFRP in the fibre direction (the 1-direction), it tends to shrink more than the carbon fibre; see Table 1. This behaviour corresponds to what we observe in our arch-shaped device.

Table 1. Materials properties. CFRP (T700 fiber type) in 1- and 2-direction indicate longitudinal and transversal to fibers direction, respectively [17-20].

\begin{tabular}{lll}
\hline Material & CTE $\left(10^{-6} \mathrm{~K}^{-1}\right)$ & $\begin{array}{l}\text { Young's modulus E } \\
(\mathrm{GPa})\end{array}$ \\
\hline Copper & 16.7 & 117 \\
Kapton & 20 & 2.5 \\
CFRP (1-direction) & 3.1 & 150 \\
CFRP (2-direction) & 24.7 & 150 \\
\hline
\end{tabular}

Similar experiments were undertaken with polyimide to study the behaviour of the material in contact with carbon fibres. Since the Cu-CFRP was an arch-shape, the polyimide-CFRP section should be flat, to create a structure as in Fig.1. Knowing the thickness of the polyimide sheet $(50 \mu \mathrm{m})$, one layer of CFRP would have been too thin to insure the device's strength. To create a flat CFRP-polyimide sample we created a balanced composite layup with three layers of fibres at $0 \% 190^{\circ} / 0^{\circ}$ to achieve a flat CFRP-polyimide sample with sufficient CFRP thickness to achieve structural strength.

Using the same approach to estimate the curvature of the CFRPpolyimide combination, we obtained the modelled deflection of only $0.04 \mathrm{~mm}$, which agreed well with the manufactured sample which remained flat once removed from the autoclave. Initial testing of the arch shaped CFRP-Cu and flat CFRP-polyimide indicated poor triboelectric harvesting when the copper and polyimide surfaces experienced contact-separation. This was thought to be due to the fact that conduction carbon fibres in the CFRP could be in direct contact with the copper and the polyimide, which would affect the results by creating a short circuit. As a result, an insulating 'peel-ply' (PP) layer was inserted between the CFRP and both the triboelectric copper or polyimide layers; see Fig.4. Peel ply is a porous fabric which plays the role of an insulator allowing the epoxy resin to adhere to the copper and polyimide materials but avoiding the carbon fibres from making direct contact with them.

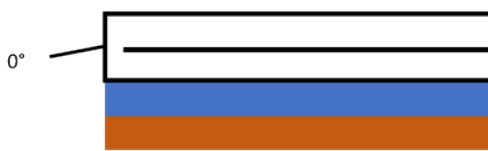

$\operatorname{CFRP}(0,2 \mathrm{~mm})$

(a)

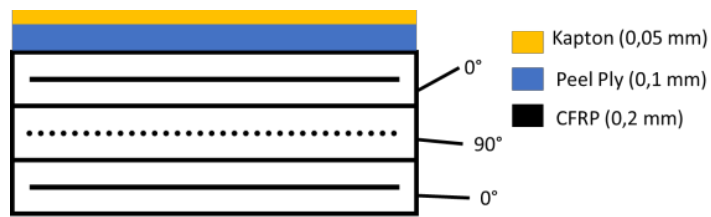

(b)

Figure 4. Schematic of samples with peel ply. (a) Top Cu-CFRP layer. (b) Bottom CFRP-polyimide layer.

Study of the effect of triboelectric response of triboelectric-CFRP combination

Fig. 5 shows the open circuit output voltage collected from contactseparation of the CFRP/PP/copper and CFRP/PP/polyimide components. We observe that the voltage maximum value is approximately $25 \mathrm{mV}$ this is less than the value obtained for the control flat samples, due to difference in the contact area. For the flat samples, the contact area was $64 \mathrm{~cm}^{2}$. While for the composites samples $3 \mathrm{~cm}^{2}$; indicating a similar voltage per unit area and indicating that the high temperature processing of the composite had not significantly affected the performance of the triboelectric layers. Theoretical triboelectric nanogenerators studies found that the output voltage was a function of the contact area $[14,21]$ given by:

$$
V=-\frac{Q}{S \cdot \varepsilon_{0}}\left(d_{0}+x(t)\right)+\frac{\sigma \cdot x(t)}{\varepsilon_{0}}
$$

With $Q$ the transferred charge, $S$ the triboelectric surface area, $x(t)$ the time-dependence distance between the two triboelectric materials, $\varepsilon_{0}$ the permittivity of free-space and $d_{0}$ the effective dielectric thickness. 


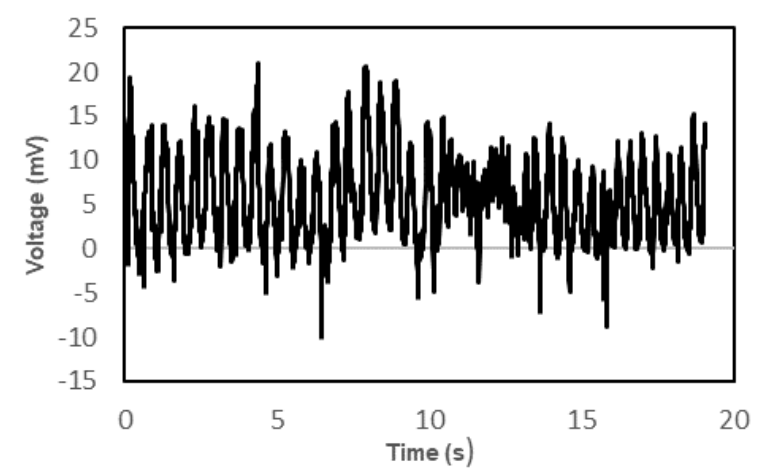

Figure 5. Open-circuit voltage obtained with the CFRP insulated samples.

\section{Observation of the bonding between materials.}

To be ensure the triboelectric materials and the CFRP composite were adhered well together, cross sections for the CFRP/PP/Cu and CFRP/PP/polyimide were prepared for optical microscopy which can be seen in Fig.6, with good bonding between the individual materials.
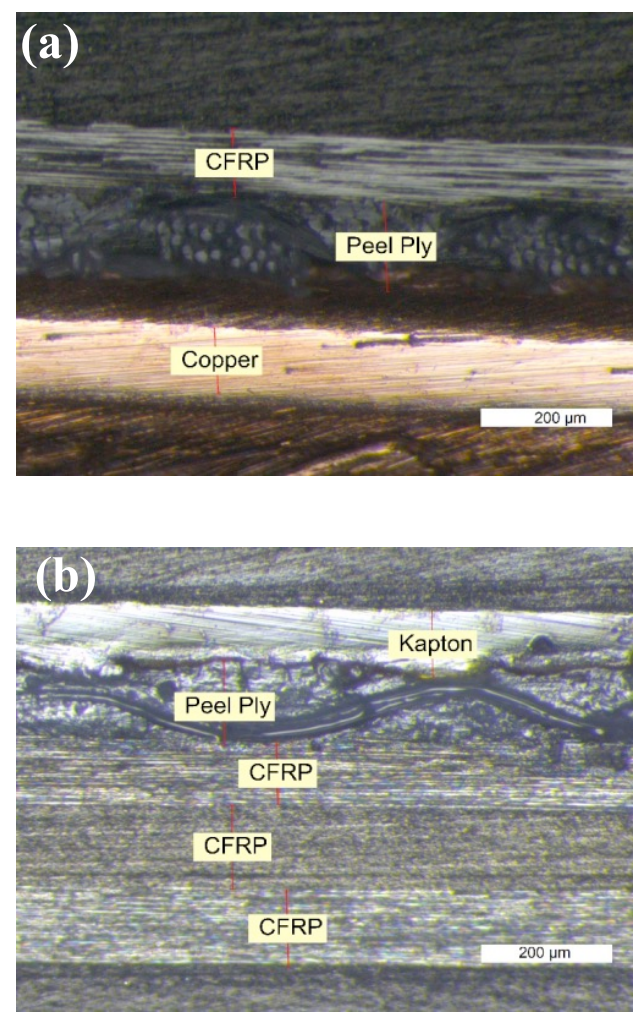

Figure 6. Optical microscopy images of the composite. (a) Cu-PP-CFRP layer b) CFRP-PP-polyimide layer.

Once the triboelectric performance of the copper and polyimide integrated with CFRP was confirmed and the correct bounding of the different layers together were confirmed it was then of interest to consider the design and manufacture of a one-piece CFRP triboelectric device. This would demonstrate the potential triboelectric CFRP structures.

\section{Design and manufacture of the single piece CFRP-triboelectric device}
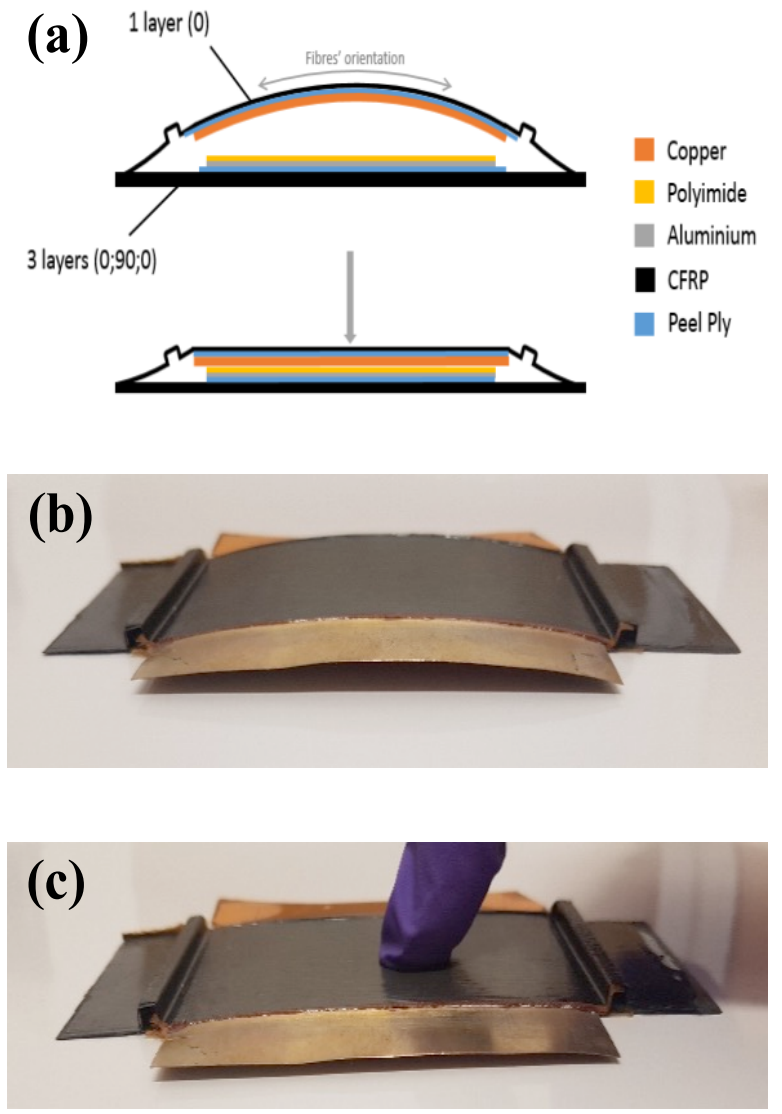

Figure 7. (a) Schematic of the one-piece triboelectric device. (b) Triboelectric device in opened position. (c) In closed position.

To create this specific shape, we used silicon rubber stripes for their ability to resist in high temperatures. In this way, the device has been put in the autoclave for 2 hours at $180^{\circ} \mathrm{C}$ and a pressure of 7 bar. The final device can be seen on figure 6.b) and c). The one-piece CFRP-triboelectric device has been designed on the basis of the integration of the individual components in Fig.67

(i) a lower planar layer of three layers of carbon fibers with a balanced lay-up $\left(0^{\circ} / 90^{\circ} / 0^{\circ}\right)$ to maintain a flat shape, peel ply for insulation and polyimide/Al

(ii) An upper layer of copper, peel ply and a single layer of carbon fibers to exploit the anisotropic thermal expansion of CFRP to create an arch shaped to layer

(iii) Carbon fibre veins on each side of the triboelectric electrodes provide the structure with flexibility to 
allow the copper sheet to be pressed against the polyimide without any resistance or risk of damage (Fig.6.a)

With an open circuit and manually activated, this device produced a voltage up to $300 \mathrm{mV}$, see Fig $8 a, b$. We also realised the charging of a capacitor of $0.1 \mu \mathrm{F}$ with our device, see Fig. $8 \mathrm{c}$.
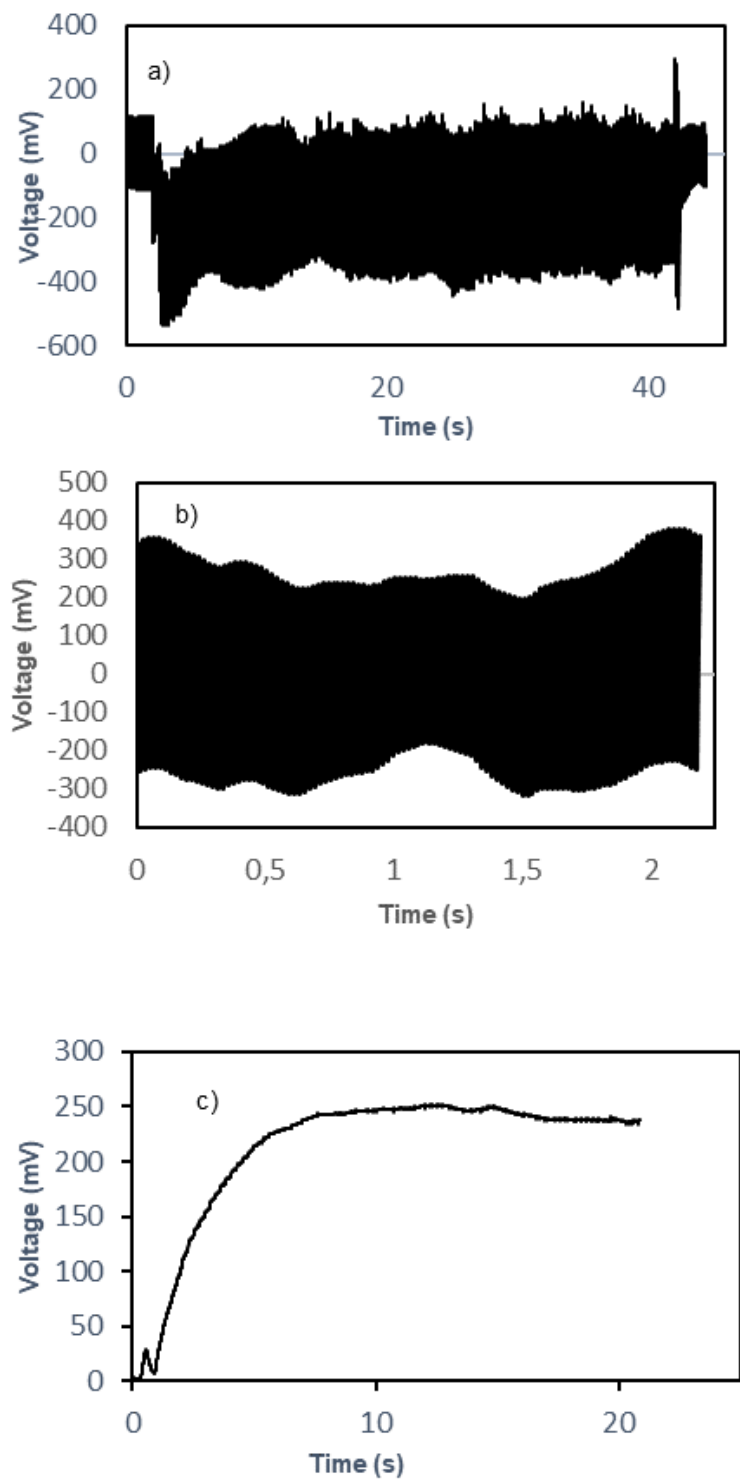

Figure 8. (a) Open circuit voltage obtained with the one-piece device. (b) Open circuit voltage in a short time scale (c) Charge of a capacitor of $0.1 \mu \mathrm{F}$.

\section{Conclusions}

We have combined structural composites and triboelectric materials to fabricate an arc-shaped hybrid carbon fiber reinforced polymer (CFRP) composite based triboelectric device. The system employs the anisotropic thermal expansion of CFRP to create a curved upper electrode made of copper. A symmetric composite architecture is used to create a flat lower polyimide electrode. This device aims to be used as a triboelectric harvesting energy source for selfpowered based structures. We have been able to manually produce a voltage up to $300 \mathrm{mV}$ which can charge a capacitor of $0.1 \mu \mathrm{F}$ to $250 \mathrm{mV}$. Further improvements conducted to a second device, similar to the first one, in which glass layers were added on the edge of copper to avoid mechanical friction between the materials. The ability to combine triboelectric and CFRP materials provides a new approach to integrate energy harvesting into engineering structures and manufacture robust devices.

\section{Acknowledgements}

Dr. Xue Yan acknowledges China Scholarship Council fund. Prof. Chris Bowen acknowledges funding from the European Research Council under ERC Grant Agreement no. 320963 on Novel Energy Materials, Engineering Science and Integrated Systems (NEMESIS).

Keywords: energy harvesting $\cdot$ triboelectric $\cdot$ arch-shaped $\bullet$ composite $\cdot$ carbon fiber

[1] A.O.Akmandor, H. Yin, N.K. Jha, "Simultaneously ensuring smartness, security, and energy efficiency in internet-of-things sensors", 2018 IEEE Custom Integrated Circuits Conference, San Diego, 2018.

[2] N. Kaur, K. Pal "Triboelectric Nanogenerators for Mechanical Energy Harvesting", Energy Technology, 2018, 6, 958.

[3] Y. J. Ko, H. S. Kim, J. H. Jung, "Arch-Shaped Triboelectric Nanogenerator as a Facile Device for Water-Wave Vibrational Energy", Journal of the Korean Physical Society, 2017, 71, 679 683.

[4] S. H. Lee, Y. H. Ko, J. S. Yu, "Facile fabrication and characterization of arch-shaped triboelectric nanogenerator with a graphite top electrode", Phys. Status Solidi A, 2015, 212, 401-405

[5] D. Kim, D-S. Kwon, Y-K. CHoi, "Self-powered fall detection system using pressure sensing triboelectric nanogenerators”, Nano Energy, 2017, 41, 139147.

[6] W. Li, J. Sun, M. Chen, "Triboelectric nanogenerator using nano-Ag ink as electrode material”, Nano Energy, 2014, 3, 95-101.

[7] G.Liu, J. Sun, M. Chen, Y. Zhao, W.Li, "The effect of anodized Ti on output performance of biomedical compatible triboelectric nanogenerators used for controlling the degradation of Mg-3wt $\% \mathrm{Zn}-0.8 \mathrm{wt} \% \mathrm{Zr}$ ", Nanotechnology, 2015, 26, 495401.

[8] M. Wang, W. Li, C. You, Q. Wang, X. Zeng, M. Chen, "Triboelectric nanogenerator based on $317 \mathrm{~L}$ stainless steel and ethyl cellulose for biomedical applications, RSC Advances, 2017, 7, 6772-6779.

[9] Y. Feng, Y. Zheng, Z. U. Rahman, D. Wang, F. Zhou, W. Liu, "Paperbased triboelectric nanogenerators and their application in self-powered anticorrosion and antifouling", J. Mat. Chem A, 2016, 4, 18022-18030.

[10] Y. Wang, Y. Yang and Z.L. Wang, "Triboelectric nanogenerators as flexible power sources”, npj Flexible Electronics, 2017, 1, 10.

[11] C.R. Bowen, R.Butler, R. Jervis, H.A. Kim, A.I.T. Salo, "Morphing and Shape Control using Unsymmetrical Composites", Journal of Intelligent Material Systems and Structures, 2007, 18, 89 
[12] D.N. Betts, C.R. Bowen, H.A. Kim, N. Gathercole, C.T. Clarke, D.J. Inman "Nonlinear dynamics of a bistable piezoelectric-composite energy harvester for broadband application", Eur. Phys. J. Special Topics, 2013, 222, 1553-1562.

[13] Z. L. Wang, J. Chen, L. Lin, "Progress in triboelectric nanogenerators as a new energy technology and self-powered sensors", Energy Environ. Sci., 2015,8, 2250-2282

[14] Z. L. Wang, J. Chen, L. Lin, S. Niu, Y. Zi, "Triboelectric Nanogenerators", Springer, Switzerland, 2016.

[15] S. Timoshenko, Analysis of bi-metal thermostats, J. Opt. Soc. Am. , 1925, $11,233$.

[16] C.J. Brampton, D.N. Betts, C.R. Bowen, H.A. Kim, "Sensitivity of bistable laminates to uncertainties in material properties, geometry and environmental conditions", Composite Structures, 2013, 102, 276-286.

[17] W. Wei, H. Rongjin, H. Chuanjun, Y. Zhao, S. Li, L. Laifeng, "Cryogenic performances of T700 and T800 carbon fibre- epoxy laminates", IoP Conf. Series: Materials Science and Engineering, 2015, 102, 012016

[18] Engineering ToolBox, Coefficients of Linear Thermal Expansion (accessed 2018)

https://www.engineeringtoolbox.com/linear-expansion-coefficients-d 95.html

[19] Engineering ToolBox, Young's Modulus - Tensile and Yield Strength for common Materials (accessed 2018)

https://www.engineeringtoolbox.com/young-modulus-d 417.html

[20] Kapton: Summary of Properties, DuPont, 2017

[21] A. Gomes, C. Rodrigues, A. M. Pereira, J. Ventura, "Influence of Thickness and Contact Area on the Performance of PDMS-Based Triboelectric Nanogenerators", ArXiv, 2018, 1803:10070. 
